\title{
35. COMMISSION DE LA CONSTITUTION DES ETOILES
}

Président: M. H. N. Russell, Princeton University Observatory, Princeton, N.J., U.S.A.

Membres: MM. Atkinson, Barbier, Bethe, Chandrasekhar, Cowling, Gamow, Hoyle, Kothari, Ledoux, Lyttleton, McCrea, Milne, Pannekoek, Randers, Rosseland, Schatzman, Schwarzschild, Severny, Strömgren, Tiercy, Tuominen, von Zeipel, Wasiutynski.

The study of the internal constitution of the stars has advanced rapidly in the past few years, especially for those of the main sequence. The present summary is necessarily brief.

For a star of uniform composition, whose interior is in radiative equilibrium, with or without a convective core, the constitution can be completely determined by successive approximations by quadratures, if there are given

(a) the atomic composition,

(b) the equation of state, defining the mean molecular weight $\mu$,

(c) the equation defining the opacity for the outflowing radiation,

(d) the equation defining the rate of liberation of energy per gram.

There is now good reason to believe that most of the mass of a star consists of hydrogen and helium. In this case $\mu$ is substantially constant throughout the interior, depending on the ratio $\mathrm{H} / \mathrm{He}$ with a small addition depending substantially on the total, but not the relative, abundance of the heavy elements. The opacity, however, depends primarily upon the abundance of these, and considerably upon what they are. If only lighter atoms of the 'oxygen group' are present, the opacity is less than if the heavier atoms of the 'iron group' are in equal amount. The liberation of energy in main-sequence stars depends upon the carbon cycle, and hence upon the relative abundance of carbon and hydrogen, the density of the hydrogen, and the temperature.

A satisfactory solution for the internal constitution of a star may be made on general physical principles if the proportions by weight of hydrogen, helium, and the heavier elements are given, and also the division of the latter between the oxygen and iron groups, and the proportion of carbon present.

This makes four independent parameters. Usually, only two other observational data-radius and luminosity-are available for a star of known mass. Assuming the distribution of the heavy elements, the abundance of hydrogen and helium may be calculated. Thus Schwarzschild* found for the Sun $47 \%$ of hydrogen, 4I of helium, and a central density 79 times the mean-assuming a 'Russell mixture' of heavier elements, containing $2 \%$ of carbon. This 'mixture' was based on estimates made at a very early stage of the study of the subject, and may underestimate the abundance of the oxygen group. On the limiting assumption that the heavy atoms all belong to this group, G. Keller $\dagger$ finds for the Sun a considerably greater central density and effective polytropic index, about the same central temperature, and a higher abundance of hydrogen.

The central concentration of density may be found observationally for eclipsing binaries with apsidal motion. The values differ from star to star, but cover almost the same range as is found in the theoretical solutions. No star is yet known for which the mass, radius, luminosity and central concentration may all be found by observation.

A fairly definite solution of the problem would be in sight if the relative abundance of carbon and oxygen could be predicted: but this is the one ratio which is known to vary widely-as in stars of classes $\mathrm{M}$ and $\mathrm{N}$.

This matter has been discussed in detail, as illustrating past success and future promise. Others can be only briefly mentioned.

Whether different parts of a star are of the same composition is an important and difficult question. Within a convective region, this may be assumed; but outer layers in radiative equilibrium may represent 'unconsumed' material; and there may be an inner isothermal core, exhausted of all its hydrogen.

\footnotetext{
* Ap. J. ro4, 203, 1946.

† Columbia University (unpublished).
} 
For stars not on the main sequence the situation is far less satisfactory, except for the white dwarfs, where the theory of relativistic degeneracy appears to be adequate. For the sub-dwarfs, it is not yet certain whether they represent an outer limit of dispersion for the main sequence, or another sequence parallel to it. So few of these stars are binaries that information regarding masses, densities, etc., is almost absent. Whether these, and the outer fringe of the main sequence on the other side, are explicable by the same physical process as for the main sequence, operating under different conditions, remains to be determined.

Very little is known about the constitution of giant stars, except that the relation between their masses and luminosities is roughly* the same as for the main sequence. The most serious lack is of information regarding the central condensation; and there is little present hope of finding this $\dagger$ by observation.

Energy can be obtained in these stars from the carbon cycle only if the central concentration is very great. No satisfactory physical theory of this has yet been developed in detail; nor has the possibility been disproved. With moderate concentration, the only known source of energy arises from irreversible transformation of light nuclei ( $\mathrm{Li}, \mathrm{Be}, \mathrm{B})$. It is hard to see how this could give the steady increase in the bolometric luminosity of normal giants from $\mathrm{G} 5$ to $\mathrm{M}$; but again the matter is not closed. The super-giants present the problem in an aggravated form.

Space fails for more than mention of the fascinating cosmogonic problems: why rotating stars have magnetic fields; what happens to a massive main-sequence star as it exhausts its hydrogen; why any super-giants remain in a universe more than $2 \times 10^{9}$ years old; and why any stars have yet reached the white-dwarf stage. There is abundant opportunity for theoretical investigation and urgent need of observation of stars which may provide critical information.

Informal and friendly co-operation among investigators appears to be all the organization which is required.

The writer is greatly indebted to Prof. Martin Schwarzschild for valuable discussions.

\section{H. N. RUSSELL \\ President of the Commission}

* The observational data for masses are rather weak.

$\dagger$ Apsidal motion must be present in $\zeta$ Aurigae- - with a period of many thousand years.

\section{Report of meeting}

Acting President: Prof. E. A. Milne.

Secretary: Dr F. Hoyle.

The President's report was discussed and approved.

It was agreed that a reprint of the B.A. Tables of the Emden functions would be most welcome, possibly with the addition of tables of solutions possessing central singularities.

Several members gave short accounts of recent work on stellar constitution. There was general agreement that models involving discontinuities of molecular weight can be chosen so as to give red giant properties. The desirability of fitting such models into a satisfactory scheme of stellar evolution was emphasized. 